Note

\title{
Ferulic Acid and Ethyl Ferulate in Sake: Comparison of Levels between Sake and
}

\section{Mirin and Analysis of Their Sensory Properties}

\author{
Katsumi Hashizume $^{1 *}$, Toshihiko Iто ${ }^{1}$, Misako Shimoнashi $^{1}$, Takahiro IshizukA ${ }^{1}$ and Masaki OкudA ${ }^{2}$ \\ ${ }^{1}$ Department of Biological Resource Sciences, Akita Prefectural University, Nakano Shimoshinjyo, Akita 010-0195, Japan \\ ${ }^{2}$ National Research Institute of Brewing, 3-7-1 Kagamiyama, Higashi-hiroshima 739-0046, Japan
}

Received October 11, 2012; Accepted March 31, 2013

Ferulic acid (FA) and ethyl ferulate (EF) in sake and mirin samples were quantified. Concentrations of FA and EF in the sake and mirin samples showed high correlations $(r=0.91$ and 0.89 , respectively). The highest level of EF in the sake samples was ca. 14-fold that of the mirin samples. Thresholds of FA and EF in a sake sample were estimated using a pipette method as $0.075 \mathrm{mg} / \mathrm{L}$ and $0.39 \mathrm{mg} / \mathrm{L}$, respectively, by eight assessors in their twenties. The FA threshold was far lower than the highest level of FA in the sake samples, which suggested that FA might affect the sensory quality of sake. FA added to the sake sample showed unpleasant bitter, astringent, "egumi", or irritating taste characteristics. Sensory and instrumental analyses suggested that EF has the ability to mitigate the taste of FA in sake.

Keywords: ferulic acid, ethyl ferulate, sake, taste, threshold

\section{Introduction}

Ferulic acid (FA) is recognized as a potent antioxidant of sake (Ohta et al., 1992) and is also known as a taste-active compound of sake. Sensory properties of FA in sake, including the threshold value in a $15 \%$ ethanol aqueous solution, were reported by Kitagaki et al. (1999). A favorable level of FA in alcoholic beverages made from rice grains was determined to be less than 10 ppm by Okamoto et al. (2007), because of its unpleasant bitter and/or astringent taste. Ethyl ferulate (EF) was reported as a key flavor compound in $m i$ rin; the Japanese traditional cooking liquor (Morita, 1975). Morita (1975) regarded FA as the precursor of EF in mirin. EF appears to be a noteworthy compound, not only because of its flavor character, but also its antioxidative or functional properties (Kikuzaki et al., 2002; Scapagnini et al., 2004). Our previous analysis on the hydrophobic taste-active compounds in sake revealed that FA and EF might contribute to the sensory quality of sake (Hashizume et al., 2012).

Hydrophobic compounds in sake, such as FA and EF, aromatic alcohols (Ito et al., 2009), and bitter-tasting peptides (Hashizume et al., 2007a; 2007b) are usually removed

*To whom correspondence should be addressed.

E-mail: hashizume@akita-pu.ac.jp by refining with charcoal during sake manufacturing (Hashizume et al., 2012). While charcoal refining is effective for removing unpleasant taste compounds, experience has shown that it also affects the agreeable taste, diminishing the taste harmony of sake. Some sake products, usually made from highly polished rice grains, such as daiginjyo-type sake, are therefore often produced without charcoal treatment.

Previous studies suggested that FA and EF, which is a key flavor compound of mirin, might play important roles in the sensory quality of sake; however, levels of FA and EF in sake and mirin have not been thoroughly documented. Furthermore, the literature on the threshold value of FA in sake has not been in good agreement, and reports on the sensory properties of EF in sake are very few. Hence, we analyzed the levels of FA and EF in sake and mirin samples, and investigated their sensory properties in sake.

\section{Materials and Methods}

Materials 4-Hydroxy-3-methoxycinnamic acid [ferulic acid (FA)], ethyl 4-hydroxy-3-methoxycinnamate [ethyl ferulate (EF)], 2-(1H-indol-3-yl) ethanol (tryptophol), and aspartic acid were obtained from Wako Pure Chemical Industries (Osaka, Japan). Sake and mirin products and a neutral spirit (Korui-shochu 20-do, Takara Shuzo, Kyoto, Japan) 
were purchased from a local market, except for sake sample (a) in Table 1. Sake sample (a) was brewed in our laboratory without charcoal treatment. Sake samples (b) and (c) were designated on the labels as charcoal-untreated products. The other sake samples and all mirin samples had no information about charcoal-treatment on the labels.

Analysis of sake and mirin samples Concentrations of FA and EF in the samples were determined by RP-HPLC as reported previously (Hashizume et al., 2012). Ethanol and sake meter were measured by an Alcomate AL-2 (Riken Keiki, Tokyo, Japan) and a DA-105 meter (Kyoto Electronics MFG., Kyoto, Japan), respectively. Extract, acidity, and amino acidity were analyzed according to the reference (The Brewing Society of Japan, 1993). Tyrosol, 2-phenylethanol, and tryptophol were determined by the method previously reported (Ito et al., 2009).

Sensory analysis Deionized and distilled water, 15\% (v/v) ethanol aqueous solution, and two commercial sake samples, (A) and (B), were used as solvents in the sensory analysis. Sake samples (A) and (B) were samples (j) and (g) in Table 1, respectively. The $15 \%$ ethanol aqueous solution was prepared from the commercial neutral spirit and water. Ingredient materials of sake sample (A) were rice, water and a neutral spirit. The sample had the following analytical data: ethanol, $14.0 \%(\mathrm{v} / \mathrm{v})$; sake meter, +1.7 ; extract, $4.5 \%(\mathrm{w} /$ v); acidity, $1.5 \mathrm{~mL}$; amino acidity, $1.0 \mathrm{~mL}$; tyrosol, $37 \mathrm{mg} / \mathrm{L}$; 2-phenylethanol, $14 \mathrm{mg} / \mathrm{L}$; tryptophol, $0.2 \mathrm{mg} / \mathrm{L} ; \mathrm{FA}, 0.0 \mathrm{mg} /$ $\mathrm{L}$; and EF, $0.00 \mathrm{mg} / \mathrm{L}$. Sake sample (B) was a jyunmai-type made from $70 \%$ polished rice grains. It showed the following analytical data: ethanol, $15.1 \%(\mathrm{v} / \mathrm{v})$; sake meter, + 3.1; extract, $4.6 \%(\mathrm{w} / \mathrm{v})$; acidity, $1.3 \mathrm{~mL}$; amino acidity, $1.5 \mathrm{~mL}$, tyrosol, $102 \mathrm{mg} / \mathrm{L}$; 2-phenylethanol, $68 \mathrm{mg} / \mathrm{L}$; tryptophol, $0.4 \mathrm{mg} / \mathrm{L}$; FA, $0.0 \mathrm{mg} / \mathrm{L}$; and EF, $0.00 \mathrm{mg} / \mathrm{L}$. Assessors were comprised of undergraduate students in their twenties in our school who were trained using a sensory test to discriminate the fundamental five tastes (Furukawa, 2001), and a teacher in his forties. Two methods were employed for sensory tests: a) the assessor dropped $200 \mu \mathrm{L}$ of a sample onto the tongue with a pipette, then tasted it (pipette method); b) the assessor tasted a sample using a traditional tasting cup for sake, Jyanome-choko (common method).

Preliminary test for assessment of threshold FA, EF, tryptophol, or aspartic acid was sequentially diluted 2-fold (1:1) with water. FA and EF were also diluted with $15 \%$ ethanol aqueous solution or the sake sample (A). The serial dilutions of each were presented to assessors in order of ascending concentration. Assessments were made by five student assessors using the pipette method.

Assessment of threshold After the preliminary test, threshold of FA or EF was examined according to the Brew-
Table 1. Concentration of ferulic acid and ethyl ferulata in sake and mirin samples.

\begin{tabular}{|c|c|c|c|}
\hline \multirow{2}{*}{ Samples } & \multirow{2}{*}{ Type } & Ferulic acid & Ethyl ferulate \\
\hline & & \multicolumn{2}{|c|}{ Concentration $(\mathrm{mg} / \mathrm{L})^{\mathrm{a}}$} \\
\hline \multicolumn{4}{|l|}{ Sake $^{b}$} \\
\hline (a) & Jyunmai $^{\mathrm{c}}$ & $4.8 \pm 0.2$ & $0.98 \pm 0.02$ \\
\hline (b) & Jyunmai ${ }^{\mathrm{c}}$ & $3.7 \pm 0.1$ & $0.86 \pm 0.01$ \\
\hline (c) & Jyunmai ${ }^{\mathrm{c}}$ & $2.3 \pm 0.1$ & $0.90 \pm 0.00$ \\
\hline (d) & Jyunmai & $1.4 \pm 0.0$ & $0.08 \pm 0.00$ \\
\hline (e) & Jyunmai & $1.3 \pm 0.0$ & $0.09 \pm 0.01$ \\
\hline (f) & Jyunmai & $0.6 \pm 0.0$ & $0.34 \pm 0.01$ \\
\hline (g) & Jyunmai & $0.0 \pm 0.0$ & $0.00 \pm 0.00$ \\
\hline (h) & Ordinary A & $0.1 \pm 0.0$ & $0.00 \pm 0.00$ \\
\hline (i) & Ordinary A & $0.0 \pm 0.0$ & $0.00 \pm 0.00$ \\
\hline (j) & Ordinary A & $0.0 \pm 0.0$ & $0.00 \pm 0.00$ \\
\hline (k) & Ordinary B & $0.2 \pm 0.0$ & $0.01 \pm 0.00$ \\
\hline (1) & Ordinary B & $0.0 \pm 0.0$ & $0.00 \pm 0.00$ \\
\hline \multicolumn{4}{|l|}{ Mirin $^{\mathrm{d}}$} \\
\hline (a) & Jyunmai & $6.5 \pm 0.4$ & $0.07 \pm 0.01$ \\
\hline (b) & Jyunmai & $2.0 \pm 0.3$ & $0.01 \pm 0.01$ \\
\hline (c) & Ordinary & $4.1 \pm 0.1$ & $0.02 \pm 0.00$ \\
\hline (d) & Ordinary & $3.0 \pm 0.2$ & $0.05 \pm 0.00$ \\
\hline (e) & Ordinary & $0.6 \pm 0.1$ & $0.00 \pm 0.00$ \\
\hline
\end{tabular}

${ }^{a}$ Data are means \pm the S.D. of three measurements.

${ }^{\mathrm{b}}$ Raw materials of sake samples were as follows: Jyunmai, rice, rice koji, and water; Ordinary A, rice, rice koji, neutral spirit, and water; Ordinary B, rice, rice koji, neutral spirit, sugar, organic acids, and water.

${ }^{\mathrm{c}}$ Samples were charcoal-untreated types.

${ }^{\mathrm{d}}$ Raw materials of mirin samples were as follows: Jyunmai, rice, rice koji, neutral spirit; Ordinary, rice, rice koji, neutral spirit, and sugar.

ery Convention of Japan sensory analysis method (Brewery Convention of Japan, 2002). This method is founded on the forced-choice ascending concentration series method of limits (ASTM, 1979). We presented 4 sets of triangular tests, though the official method demanded 6 sets, to prevent sensory fatigue of the assessor. If the threshold was not found in the presented test set, another set of triangular tests, which included the lower or the higher level, was presented to the assessor. Assessments were conducted by eight student assessors using the pipette method.

Effects of EF on sensory properties of FA added to the sake sample The triangular tests were assessed by five assessors, consisting of four students and one teacher, using the common method. The triangular set of test (I) consisted of sake (B) and of sake (B) plus $6 \mathrm{mg} / \mathrm{L}$ FA. Test (II) consisted of sake (B) and of sake (B) plus $6 \mathrm{mg} / \mathrm{L} \mathrm{FA}$ and $1.2 \mathrm{mg} / \mathrm{L} \mathrm{EF}$. Five assessors each tested 3 sets of triangular tests for tests (I) and (II). The FA and EF added samples of tests (I) and (II) were subjected to an instrumental analysis using a SA402B taste sensor system (Insent, Kanagawa, Japan) furnished with 
five sensors for food analysis, SB2AAE (umami), SB2CT0 (salty), SB2CA0 (sour), SB2C00 (bitter), and SB2AE1 (astringent). For the sensors, SB2AAE, SB2C00, and SB2AE1, changes in membrane potential adsorption were also analyzed.

\section{Results and Discussion}

Levels of FA and EF in sake and mirin samples Concentrations of FA and EF in the sake samples $(n=12)$ ranged from nil to $4.8 \mathrm{mg} / \mathrm{L}$ and from nil to $0.98 \mathrm{mg} / \mathrm{L}$, respectively. In the mirin samples, FA ranged from 0.6 to $6.5 \mathrm{mg} / \mathrm{L}$ and $\mathrm{EF}$ ranged from nil to $0.07 \mathrm{mg} / \mathrm{L}$ (Table 1). Charcoal-untreated jyunmai-type sake samples (a), (b), and (c) showed high FA and EF levels, while jyunmai-type sake samples (d), (e), and (f) showed rather low FA and EF levels. Differences might be due to a light charcoal treatment, for example 100-500 $\mathrm{mg} / \mathrm{L}$, of the samples in the refining process. The other sake samples showed very low or undetectable levels of FA and EF, which might be due to a heavy charcoal treatment, for example more than $1000 \mathrm{mg} / \mathrm{L}$. The reason for the heavy charcoal treatment might be that ordinary-type and some jyunmai-type sake made from low grade white rice grains usually contain high levels of unpleasant bitter and astringent compounds immediately after brewing. It is proposed that the factor with the greatest influence on the variation of FA and EF levels in sake is the charcoal treatment; however, other factor such as the volume of exogenous alcohol addition, or polishing grade of the ingredient rice grains may also play a role. EF has been thought to be an important flavor compound of mirin (Morita, 1975); however, all mirin samples showed low levels. The observed low EF level might reflect the peculiar conditions of the mirin production process, since charcoal treatment is not indispensable for the mirin refining process (Kawabe and Morita, 1998). The highest level of EF in the sake sample was ca. 14-fold higher than that of the mirin sample. High correlations were observed between the level of FA and EF in both sake $(r=0.91)$ and mirin $(r=0.89)$, though the ratio of EF/FA in sake was ca. 25 -fold higher than that in mirin. Although the use of rice koji and steamed rice grains as ingredient materials is common to both sake brewing and mirin production, alcoholic fermentation does not occur in the mirin mash but does occur in the sake mash. Therefore, the formation of FA and EF might be due to the common ingredient materials, whereas difference in the ratio of EF/FA might be due to factors derived from the differences in manufacturing conditions between sake brewing and mirin production.

\section{The threshold of FA or EF added to the sake sample} Before a systematic examination of threshold, concentrations for detection and recognition of FA, EF, tryptophol,

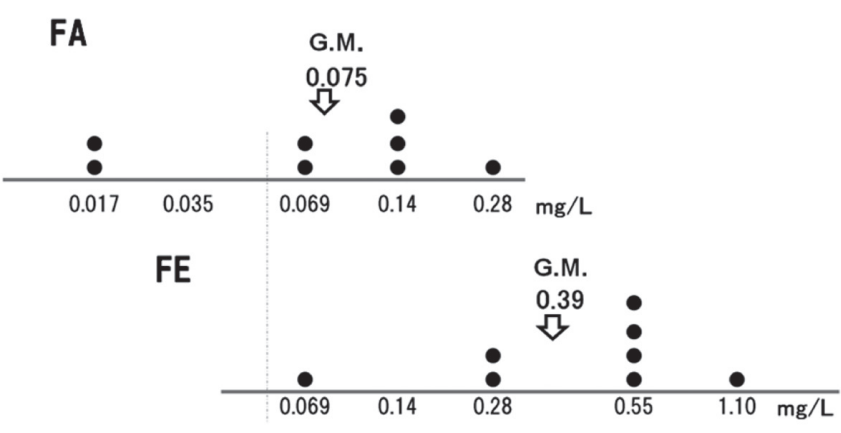

Fig. 1. Frequency of estimated thresholds for eight assessors.

G.M. indicates geometric mean. indicates an assessor. Data were obtained by eight assessors using the pipette method.

and aspartic acid in water were examined. FA and EF were also examined in the $15 \%$ ethanol aqueous solution or sake sample (A) (Table 2). Sake sample (A) was used in this test because FA and EF were not detected in it and its weak flavor was deemed suitable for detecting the added compounds. Tryptophol and aspartic acid were employed to confirm the reliability of this test. Geometric means of concentrations for detection and recognition in the water showed a similar rank among the four compounds tested, although the variance was large among assessors. The levels of FA and EF were lower than that of tryptophol, which is reported as a potent unpleasant-taste compound in sake (Ito et al., 2009). The level for recognition of aspartic acid in water was similar to that of a previous report (Iwano et al., 2004). Taste characteristics described by assessors for FA and EF in water were similar to our previous report (Hashizume et al., 2012). Among the three solvents tested, concentrations for detection and recognition of FA did not show a distinct difference. On the other hand, those of $\mathrm{EF}$ in the $15 \%$ ethanol aqueous solution showed higher levels than those of the water or sake sample (A). This may be due to the similarity in taste characteristics of EF and ethanol.

The threshold value for FA or EF in sake sample (A) obtained in this study is shown in Fig. 1. The geometric mean value was $0.075 \mathrm{mg} / \mathrm{L}$ and $0.39 \mathrm{mg} / \mathrm{L}$ for FA and $\mathrm{EF}$, respectively. These values were higher than the recognized levels of the preliminary examination (Table 2). The obtained threshold value of FA was far lower than the previously reported threshold value of $291.5 \mathrm{mg} / \mathrm{L} 15 \%$ ethanol aqueous solution (Kitagaki et al., 1999), which was determined by a veteran sake manufacturing panelist. Differences between the two threshold values may be due to differences in panelists and/ or experimental conditions. The threshold value of this study does not contradict the level of FA for favorable acceptance in sake, which is less than $10 \mathrm{mg} / \mathrm{L}$, suggested by Okamoto et al. (2007). It is noteworthy that the young assessors gave 
Table 2. Sensory test of ferulic acid ethyl ferulate.

\begin{tabular}{|c|c|c|c|c|c|c|c|c|}
\hline \multirow{2}{*}{$\begin{array}{l}\text { Compound } \\
\text { Solvent }\end{array}$} & \multicolumn{3}{|c|}{ Ferulic acid } & \multicolumn{3}{|c|}{ Ethyl ferulate } & \multirow{2}{*}{$\begin{array}{c}\text { Tryptophol } \\
\text { Water }\end{array}$} & \multirow{2}{*}{$\frac{\text { Aspartic acid }}{\text { Water }}$} \\
\hline & Water & $15 \%$ Ethanol & Sake & Water & $15 \%$ Ethanol & Sake & & \\
\hline \multicolumn{9}{|c|}{ Detected concentration $^{\mathrm{a}}(\mathrm{mg} / \mathrm{L})$} \\
\hline Maximum & 0.098 & 0.098 & 0.098 & 0.195 & 0.390 & 0.098 & 0.098 & 200 \\
\hline Minimum & 0.002 & 0.006 & 0.003 & 0.012 & 0.024 & 0.003 & 0.024 & 12.5 \\
\hline Geometric mean for 5 & 0.008 & 0.011 & 0.011 & 0.037 & 0.074 & 0.024 & 0.043 & 33 \\
\hline \multicolumn{9}{|c|}{ Recognized concentration $^{\mathrm{b}}(\mathrm{mg} / \mathrm{L})$} \\
\hline Maximum & 0.195 & 0.195 & 0.195 & 0.195 & 0.390 & 0.195 & 0.390 & 200 \\
\hline Minimum & 0.006 & 0.006 & 0.006 & 0.049 & 0.049 & 0.003 & 0.049 & 6.3 \\
\hline Geometric mean for 5 & 0.028 & 0.021 & 0.024 & 0.074 & 0.148 & 0.064 & 0.130 & 66 \\
\hline
\end{tabular}

Data were obtained by five assessors using the pipette method.

${ }^{a}$ The concentration at which a taste stimulus was detected by assessor.

${ }^{\mathrm{b}}$ The concentration at which a taste was recognized by assessor

a lower threshold value than the veteran panelist; experimental conditions greatly influenced observed threshold values (Furukawa, 2001b). The maximum level of FA in the sake sample is far higher than the estimated threshold; therefore, FA may affect the sensory quality of sake. FA may exert a negative sensory effect because of its unpleasant bitter and/ or astringent taste. In this study, the number of assessors was not sufficient for a precise estimation of threshold (Brewery Convention of Japan, 2002); therefore, further experiments using more assessors (from 20 to 30 assessors) is desirable. The maximum level of EF in the sake was higher than the threshold, which indicated a contribution of EF to the sensory quality of sake. EF may exert a favorable effect because of its pleasant, namely sweet, taste accompanying moderate bitter and/or astringent taste, at low concentrations.

Effects of EF on the sensory properties of FA added to the sake sample Sake sample (B) was used in tests (I) and (II) because FA and EF were undetectable, and its medium flavor was deemed suitable for detecting differences in taste characteristics. The common method was used to carefully assess delicate differences in the complicated sake taste. A sake sample prepared by adding $6 \mathrm{mg} / \mathrm{L}$ FA to sake (B) was distinguished as significantly different from sake (B) through triangular test (11 correct answers in 15 trials; ${ }^{* *}$ ), while sake (B) and sake sample (B) plus $6 \mathrm{mg} / \mathrm{L} \mathrm{FA}$ and $1.2 \mathrm{mg} / \mathrm{L}$ EF (B) were not significantly different (3 correct answers in 15 trials) in test (II). On the distinguished samples in the test (I), bitter, astringent, "egumi" or irritating taste were pointed out, which might be due to the added FA. The FA and EF added samples of tests (I) and (II) were analyzed using an instrumental sensor system. Differences were observed on the output of sensors SB2AAE (umami), SB2CT0 (salty) and SB2AE1 (astringent), although the signal output of each sensor was weak and not so stable (Fig. 2). Prior to this instrumental analysis, we confirmed that quinine and tyrosol

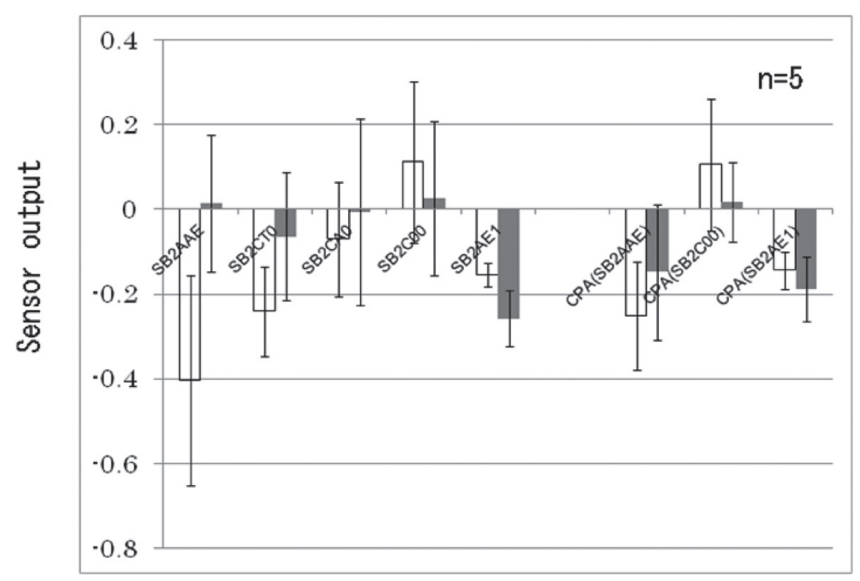

Fig. 2. Results of the analysis for the FA and EF added samples of tests (I) and (II) by the taste sensory system. $\square, 6 \mathrm{mg} / \mathrm{L} \mathrm{FA}$;, 6 $\mathrm{mg} / \mathrm{L} \mathrm{FA}$ and $1.2 \mathrm{mg} / \mathrm{L} \mathrm{EF}$. Data are expressed as means \pm S.D. for five measurements.

$(1 \mathrm{mM})$ in the sake, well-known bitter compounds, produced distinct responses in the sensors SB2AAE (umami) and SB2CT0 (salty). In contrast, these bitter compounds scarcely produced responses in SB2C00 (bitter). FA (100 mg/L) in the sake showed a similar response to quinine and tyrosol (data not shown). The effect of EF observed in this analysis, namely decreasing the signal output of SB2AAE and SB2CT0 sensors, might therefore be correlated with the results of the human sensory examination in this study.

From the sensory and instrumental analyses, it was indicated that $\mathrm{EF}$ at $1 / 5$ concentration of FA mitigates the taste of FA in the sake. The masking effect of EF to FA in the sake is very noteworthy, since the taste of FA is not favorable. The effect of EF might contribute to the observation that the charcoal-untreated sake with a high EF level had minimal taste disharmony in spite of the high FA level (Hashizume et al., 2012). It is thought that the structural similarity, as well as minor variations, of both compounds is related to this 
observation. The effect of EF on FA in sake requires further investigation with respect to the EF to FA ratio.

Acknowledgement We are grateful to the students of the Food and Brewing Group, Department of Biological Resource Sciences of Akita Prefectural University for their assistance with the sensory analysis.

\section{References}

ASTM E679. (1979). Standard practice for determination of odor and taste threshold by a forced-choice ascending concentration series method of limits. Philadelphia, PA.

Brewery Convention of Japan. (2002). "BCOJ Kanno Hyoka Ho." The Brewing Society of Japan, Tokyo (in Japanese).

Furukawa, H. (2001a). “Oishisa wo Hakaru.” Saiwai Shobo, Tokyo, pp.7-8 (in Japanese).

Furukawa, H. (2001b). “Oishisa wo Hakaru.” Saiwai Shobo, Tokyo, pp.68-69 (in Japanese).

Hashizume, K., Okuda, M., Numata, M. and Iwashita, K. (2007a). Bitter-tasting sake peptides derived from the N-terminus of the rice glutelin acidic subunit. Food, Sci. Technol. Res., 13, 270-274.

Hashizume, K., Okuda, M., Numata, M., Zhou, Y. and Koseki, T. (2007b). Characterization of peptides generated in proteolytic digest of steamed rice grains by sake koji enzymes. J. Biosci. Bioeng., 104, 251-256.

Hashizume, K., Ito, T., Shimohashi, M., Kokita, A., Tokiwano, T. and Okuda, M. (2012). Taste-guided fractionation and instrumental analysis of hydrophobic compounds in sake. Biosci. Biotechnol. Biochem., 76, 1291-1295.
Ito, T., Komatsu, Y., Takado, A., Takahashi, H., Tamogami, S., Koizumi, T., Nakazawa, N. and Iwano, K. (2009). Tastes of the aromatic alcohols in Ginjyo-syu. J. Brew. Soc. Japan, 103, 562569 (in Japanese).

Iwano, K., Takahashi, K., Ito, T. and Nakazawa N. (2004). Search for amino acids affecting the taste of Japanese sake, J. Brew. Soc. Japan, 99, 659-664 (in Japanese).

Kawabe, T. and Morita, H. (1998). J. Brew. Soc. Japan, 93, 799-806 (in Japanese).

Kikuzaki, H., Hisamoto, H., Hirose, K., Akiyama, K. and Taniguchi, H. (2002). Antioxidant properties of ferulic acid and its related compounds. J. Agirc. Food. Chem., 50, 2161-2168.

Kitagaki, H., Iwatsuki, Y. and Tsugawa, M. (1999). Sensory evaluation of a model sake endowed with free radical scavenging ability. J. Brew. Soc. Japan, 94, 502-506 (in Japanese).

Morita, H. (1975). Kagaku to Seibutsu, 2, 96-104 (in Japanese).

Ohta, T., Takashita, H., Todoroki, K., Iwano, K. and Ohba, T. (1992). Antioxidative substances in sake. J. Brew. Soc. Japan, 87, 922926 (in Japanese).

Okamoto, M., Kakimoto, T. and Ito, K. (2007). Japan Kokai Tokkyo Koho, TokuKai 2007-202504. Aug. 16.

Scapagnini, G., Butterfield, D.A., Colombrita, C., Sultana, R., Pascale, A. and Calabrese, V. (2004). Ethyl ferulate, a lipophilic polyphenol induces HO-1 and protects rat neurons against oxidative stress. Antioxdants \& Redox Signaling, 6, 811-818.

The Brewing Society of Japan (1993). "Official methods of analysis of National Tax Administration Agency, $4^{\text {th }}$ ed." The Brewing Society of Japan, Tokyo, pp.20-27 (in Japanese). 